\title{
Design and Application of Pseudorandom Coded Sweep for Mini- SOSIE
}

\author{
Chen Zubin ${ }^{a, b}$, Li Na $^{a}$, Long Yun ${ }^{a}$, Wen Xiaozhe ${ }^{a}$, and Sun Feng ${ }^{a, b^{*}}$ \\ ${ }^{a}$ College of Instrument Science and Electrical Engineering, Jilin University; \\ ${ }^{\mathrm{b}}$ Key Lab of Geo-Exploration and Instrumentation, Ministry of Education, Changchun, \\ China \\ *Corresponding author: Sun Feng, sunfeng@jlu.edu.cn
}

\begin{abstract}
The miniaturized, high-energy-output mini-SOSIE has become an important source in seismic exploration. It has seriously impeded resolution because of the coherent vibration frequency and periodical problem of the signal. In order to solve these problems, this paper designed a vibrator signal generator. In this paper, a pseudorandom coding technique is tested as a sweep control method for mini-SOSIE. The pseudorandom coding sequence is produced by Matlab and is of good auctocorrelation characteristic. By comparing experimental field data from sources which is controlled by pseudorandom coding method and the rammer, the pseudorandom scheme yields significant improvements, and greatly improved signal-to-noise (SNR) and resolution.
\end{abstract}

Key words: seismic, mini-SOSIE, pseudorandom, SNR, resolution

\section{Introduction}

The requirements of seismic exploration are difficult to meet with vibrator source driven by electromagnetic and hydraulic, because its mobility and power are hampered by complex topography in the field. The miniaturized, high-energy-output mini-SOSIE has recently drawn increasing attention, because it can circumvent these problems. ${ }^{[1,2]}$ Barbier first proposed mini-SOSIE seismic exploration with a random method, in which varying the engine speed changes the strike rate, resulting in randomly timed impulses. ${ }^{[3]}$ But there are serious interferences because of the rammer's periodical operation, and this hampered seismic resolution, thus reduced data's SNR. However, pseudorandom vibroseis sweeps have long been suggested as an alternative to standard linear sweep due to their potential for using superior orthogonality, ${ }^{[4,5]}$ a lower likelihood for damaging infrastructure and increasing lowfrequency content and pseudorandom signals are attractive as they have a better autocorrelation shape, they have the ability to reduce interference noise. ${ }^{[6,7]}$ Nasreddin et al applied pseudorandom signal coding to simultaneous vibroseis data, ${ }^{[8]}$ which resulted in improving data quality and greater sampling efficiency. Sallas et al and Maxwell et al applied similar pseudorandom coding to low-frequency vibroseis data, which resulted in improvements to temporal resolution. It provides a theoretical basis to improve the resolution and signal-to-noise ratio of seismic signal. ${ }^{[9,10]}$

This paper mainly introduces a pseudorandom coded sweep control method for mini-SOSIE. We study the principles of the pseudorandom coding and the characteristics of the resultant coding sequences. Tests on synthetic data suggest that a pseudorandom coding scheme outperforms comparable schemes based on the rammer working alone. 


\section{Mini-SOSIE signal analyses}

For mini-SOSIE, the battering ram is used as the seismic source, so its structure, performance and signal characteristics will directly affect the seismic exploration results. The battering ram is equipped with high strength spring in its intermediate position, it can do up and down reciprocating motion and generate impact.

In order to have a better understanding of the signal characteristic of mini-SOSIE, we collect continuous signal on the base plate of the battering ram as is shown in Fig 2.1. Fig 2.1(a) is data sampled at $1 \mathrm{~ms}$, Fig 2.1 (b) is the autocorrelation result; Fig 2.1(c) is the analysis of the seismic section spectrum. From Fig 2.1(b) we know that the autocorrelation result is of poor quality and there is severe interference, also the amplitude in the rear is a little high. For amplitude spectrum in Fig 2.1 (c) is discrete, which is bad for seismic exploration. Analyzing 1024 sampling points in Fig 2.2, we can conclude that the mini-SOSIE signal is periodical and is about $12 \mathrm{~Hz}$, the impact amplitude is basically identical.

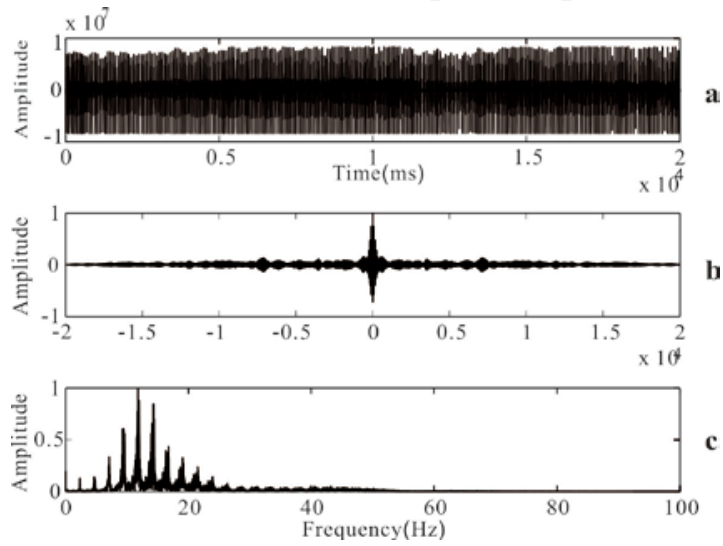

Fig 2.1 Data acquisition result: (a) is data sampled at $1 \mathrm{~ms}$ for $20000 \mathrm{~ms}$, (b) is the autocorrelation result, (c) is the analysis of the seismic section spectrum.

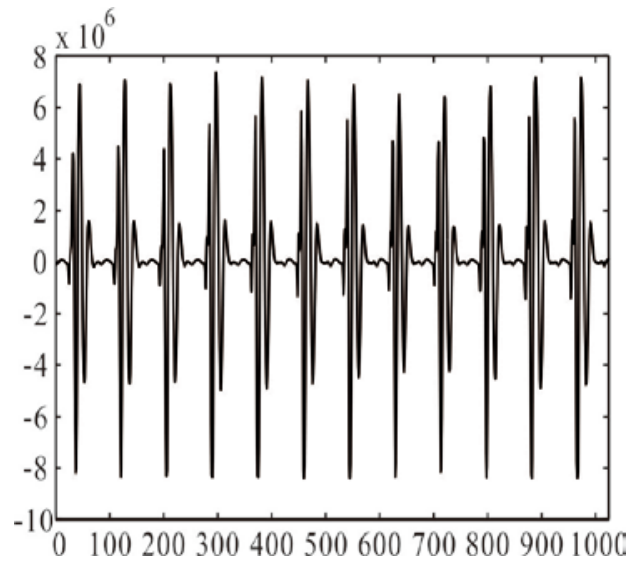

Fig 21024 sampling points

\section{Design and implementation of pseudorandom coding}

In order to get better seismic exploration result, it is necessary to make the mini-SOSIE work in a safe frequency range. However, because of the mechanical properties of mini-SOSIE itself, it cannot realize fully random vibration, thus there will be large interference. But for pseudorandom coding, it is with good autocorrelation and anti-interference characteristic, and is easy to be separate from other signals, thus it can well solve the periodical problem of miniSOSIE. ${ }^{[11]}$

\subsection{Principle of pseudorandom coding}

Pseudorandom coding sequence is composed of element 0 and 1 , here we use a linear shift register (Fig 3.1) to generate a sequence. ${ }^{[12,13]}$ 


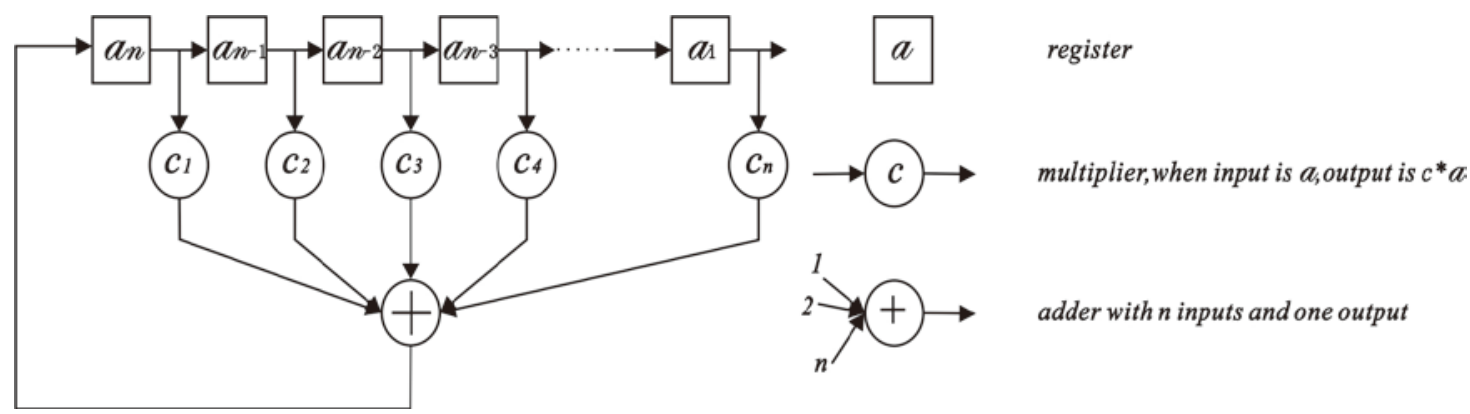

Fig 3.1 Flow diagram representation

The initial state of the shift register is $\left(a_{1}, a_{2}, \ldots a_{n}\right)$. When a shift pulse is generated, the contents of each level move to the next level, and the contents of each level are simultaneously multiplied and added. The output is represented by the filtering operation

$$
a_{n+1}=a_{1} c_{n}+a_{2} c_{n-1}+\ldots+a_{n-1} C_{2}+a_{n} c_{1}
$$

In the final stage, because output is sent to the first shift register, the state of the register is $\left(a_{2}, a_{3}, \ldots a_{n+1}\right)$, so the output is $a_{1}$. With a consistent shift pulse, the output is a $q$-ary sequence: $q=2$ yields a 2-ary sequence, after generating 2-ary sequence, it needs to do two modular arithmetic. $\mathrm{N}$ decides the order of the sequence. Fig 3.2 is a group of simulation diagram.

Fig 3.2(a) is fourth order 2-ary sequence; Fig 3.2(b) is a fourth order binary pseudorandom sequence. Fig 3.2(c) is the autocorrelation result of fourth order binary pseudorandom sequence.
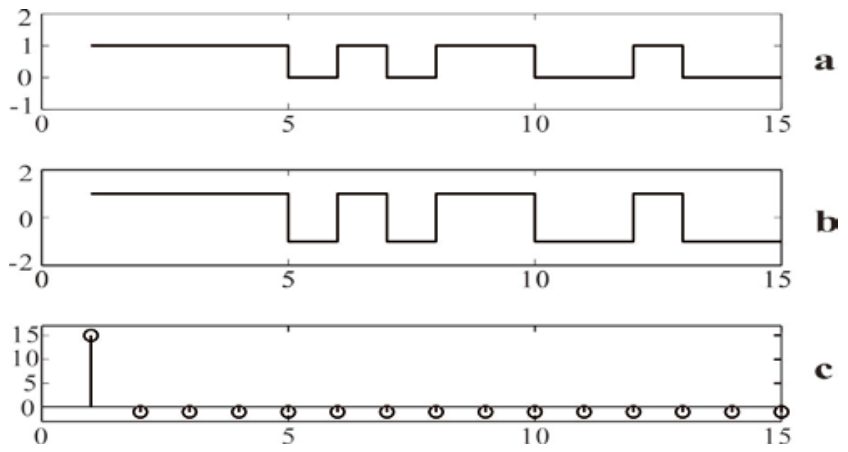

Fig 3.2is a group of simulation diagram. Fig 3.2(a) is fourth order 2-ary sequence; Fig 3.2(b) is a fourth order binary pseudorandom sequence; Fig 3.2(c) is the autocorrelation result of fourth order binary pseudorandom sequence.

Here we get a good autocorrelation result; it is similar to the characteristic of a pulse function. Thus we conclude that pseudorandom coding technique is of great research value for miniSOSIE.

\section{Results of field testing}

In order to realize pseudorandom coded sweep for mini-SOSIE, we designed a vibrator excitation signal generator, and carried out a field test to verify the consistency of theory and actual result. We used a GeometricsGEIST-96 with 24 channels and JF-20DX-10Hz sensors spaced every $2 \mathrm{~m}$. Data were sampled at $1 \mathrm{~ms}$ for $1000 \mathrm{~ms}$. Fig 4.1 is the seismic records before improvement. 


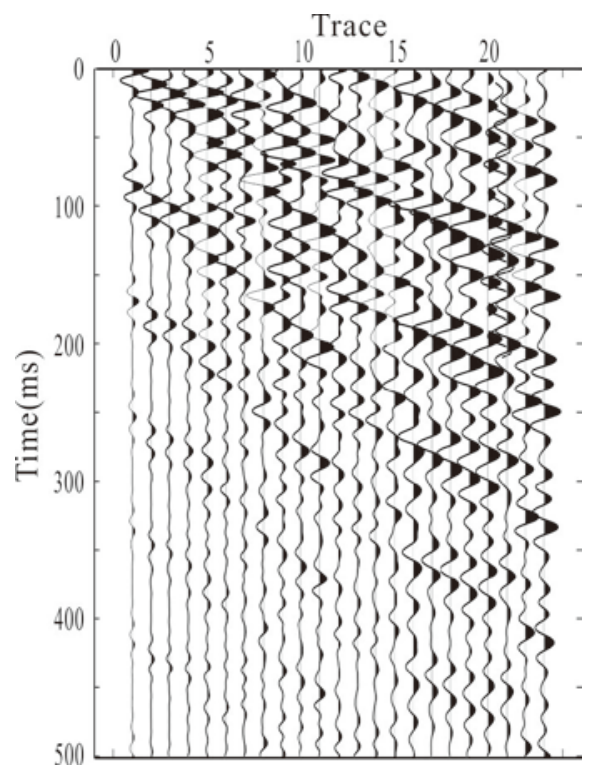

Fig 4.1 Seismic records before improvement

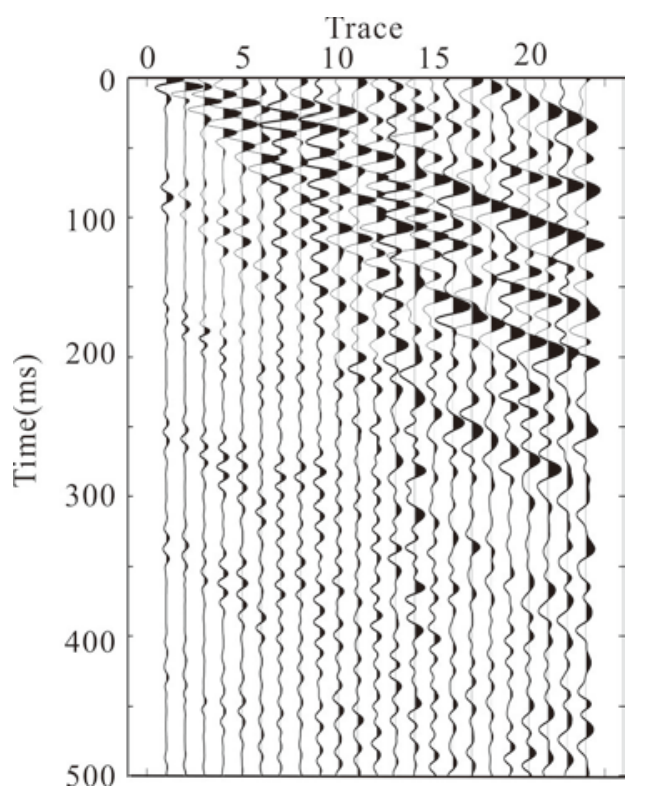

Fig 4.2 Seismic records collected by a fourth order pseudorandom coding method

Fig 4.2 Seismic records collected by a fourth order pseudorandom coding method Comparing the both records, we can conclude that the seismic records generated by a fourth order pseudorandom coding method have higher temporal resolution, and can suppress surface waves, improves resolution, reduce false reflections and coherent noise significantly, greatly improved SNR and resolution.

\section{Conclusions}

Mini-SOSIE is more and more applied to the seismic exploration conditions are susceptible to constrain environment because it is portable and easy to carry. Resolution and SNR are the major factors influencing seismic exploration, and there exists periodic and interference for the original mini-SOSIE and seriously impeded the seismic exploration effect. This paper, we proposed a pseudorandom coded sweep scheme for the mini-SOSIE, and designed an excitation signal generator. Field tests showed our method yields a higher SNR with fewer false reflections and higher resolution, making it suitable for shallow seismic exploration.

\section{Acknowledgements}

The research was sponsored by the Natural Science Foundation of China (No. 41404097, No. 41304139), the Science and Technology Development Project of Jilin Province (No. 20150520071JH), China Postdoctoral Science Foundation (No.2015M571366), and SinoProbe-Deep Exploration in China (SinoProbe-09-04).

\section{References}

1. X. Chang, Y. Liu, Seismic profile of the Huangzhuang-Gaoliying fault in Beijing by mini-SOSIE method, SEG Expanded Abstracts. 29 (2010) 1970-1974.

2. K. Driml, M. Reveleigh, K. Bartlett,Mini-SOSIE-successful shallow 3D seismic data acquisition in an environmentally sensitive area, ASEG 2001, Brisbane, Expanded Abstracts. (2001) 1-4. 
3. M. G. Barbire, P. Bondon, R. Mellinger, J. R.Viallix,Mini-Sosie for land Seimology. Geophysical Prospecting. 24 (1976) 518-527.

4. Dean Timothy, Establishing the limits of vibrator performance - experiments with pseudorandom sweeps, SEG Annual Meeting 2012, Las Vegas Nevada. (2012) 1-4.

5. T. Dean, The use of pseudorandom sweeps for vibroseis surveys. Geophysical Prospecting. 62 (2014) 50-74.

6. A.B.Cunninggham, Some alternate vibrator signals, Geophysics. 44(1979), 1901-1921.

7. H. Nasreddin, T. Dean, K. Iranpour, The use of pseudorandom to reduce interference noise in simultaneous Vibrosies surveys. 22nd International Geophysical Conference and Exhibition. (2012) 1-4.

8. H.Nasreddin, T. Dean, K. Iranpour, The use of pseudorandom sweeps to reduce interference noise in simultaneous Vibrosies surveys, ASEG 2012, Brisbane, Australia.(2012) 1-4.

9. S. John, G. John, M. Peter, F. Lin, Pseudorandom sweeps for simultaneous sourcing and low-frequency generation,The Leading Edge. 30 (2011) 1162-1172.

10. M.Peter, G. John, E.u Alexandre, F. Lin, B. Guido, S. John, Extending low frequency bandwidth using pseudorandom sweeps,SEG Expanded Abstracts 29(2010), 101-105.

11. S Shaun and H. Steve, Numerical modeling of Pseudo-random land seismic sources. ASEG 2004, Sydney, Extended Abstracts. (2004) 1-4.

12. A.Peinado, A. S. Fuster, Generation of pseudorandom binary sequences by means of linear feedback shift register (LFSRs) with dynamic feedback, Mathematical and Computer Modelling. 57 (2013) 2596-2604.

13. D.V.Sarwate, M. B.Pursley, Crosscorrelation properties of pseudorandom and related sequences, Proceedings of the IEEE.68 (1980) 1554. 